\title{
A Survey on different Types of Intrusion Detection Systems
}

\author{
Mayur V. Suramwar \\ Department of Computer Science and Engineering \\ SGGSIE\&T \\ Nanded - 431606 (M.S.) INDIA
}

\author{
Bansode S.M. \\ Department of Computer Science and Engineering \\ SGGSIE\&T \\ Nanded - 431606 (M.S.) INDIA
}

\begin{abstract}
Modern network systems have abundant trouble in security vulnerabilities like buffer overflow, bugs in Microsoft web, SQL injection, security of applications and operating systems, Sniffer Attack. Also, wireless devices mostly personal computers, sensors, personal digital assistants, and smart phones became economically doable as a result of advances in communication and manufacturing of small sensors. There are many kinds of different vulnerabilities to be exploited in such types of devices. Therefore to enhance different kind of securities, many kinds of mechanism are developed such as access control, cryptography, authentication, and many intrusion detection systems. Intrusion detection methods broadly organized into following two different types: one is anomaly detection and other one is misuse detection. Anomaly detection provides number of ways to try and verify whether the deviation is from the confirmed traditional usage patterns or not. The crucial fortune of anomaly detection lean on the expected pattern behaviors. Also, misuse detection system use different types of attacks which are known or different inadequate spots of the different systems to verify intrusions. The weakness of misuse detection system is not able to find any upcoming (unknown) intrusion until the system does not know the corresponding attack signatures.
\end{abstract}

\section{Keywords}

Security challenges, threat and countermeasures, anomaly detection, intrusion detection systems, misuse detection.

\section{INTRODUCTION}

The Internet become more convenient in people's lives as a result of consumers use the web for banking, shopping, entertain, and also invests on-line. Nowadays, the furnishing of different mobile devices (e.g. sensible phones, PDAs, sensors) has significantly risen by completely different varieties of connectivity such as GSM (global system for mobile communications), Bluetooth, Wi-Fi, GPRS. Moreover, e-commerce establishes numerous kinds of network services to enhance client satisfaction, promote service potency and quality, and cut down service value. Personal computer systems face the danger of unauthorized access and the destruction of service by outsiders. Therefore, it's essential for a contemporary society to establish a secure and trusted network dealing environment. Most network systems suffer from security vulnerabilities, e.g. SQL injection, bugs in Microsoft Internet explorer, sensing element network routing protocols too simple, and applications and operating systems having plenty of security flaws. In the same trend, number of vulnerabilities are progressively exploited in offensive mobile devices. Additional techniques are ready to eliminate such problems, such as software engineering, number of security policies, and correct configurations, user authentication (e.g. passwords, biometrics), access controls, firewalls and data protection (e.g. encryption and decryption). However, it's not possible to create the computer system which is more secure in difficult network environment. Therefore, intrusion detection systems become additional and more vital within the imperfect security environment. An intrusion is "a collection of actions that aspire to understand the confidentiality, integrity or accessibility of different resource". Intrusion can also be outlined as "a collection of actions conceive to acquire unauthorized resources, misuse rights, cause complete systems and networks crashed, decrease running potency, or deny services". Thus, IDS may be a system to observe events in computers or networks and analyses the protection violation of integrity and confidentiality of resources. In general, intrusion detection methods may be partition into misuse and anomaly detection. A misuse detection methodology is applied to observe attacks by comparing the network traffic and signature databases of acknowledged attacks. The imperfection of misuse detection is not able to mark new type of attacks till equivalent attack signatures are invaded into the database. Anomaly detection assume the model of traditional usage patterns like I/O activities from a long time historical profile and detects attacks by determining whether or not the deviation is from the traditional model or not. The other part of this paper is well regulated as follows. IDS tools are introduce in section II. In Section III, we present the criterions of IDS. Security attacks square measure delineated in Session IV. Intrusion detection systems square measure introduce in Section V. The IDS challenge on network trends are describe in section VI. Conclusions square measure introduced in Section VII.

\section{TYPES OF IDS TOOLS}

There are different IDS tools for calculating real time traffic inspection, packet logging, processing alert information, and monitoring felonious attempts such as Tcpdump, NetXRay, Ethereal, Snort, Bro [1], Sniffer, N2Fk, and network flight recorder [2]. Tcpdump may be a packet-encapsulate program for UNIX to produce functions to watch, diagnose, and log network traffic. WinDump is nothing but the Windows version of Tcpdump. Ethereal also assigns a network protocol analyzer to permit the examination of information from a live network. Snort is nothing but a combination of logger and sniffer, and provides a cross- program environment. It is conjointly a light- shallow intrusion detection tool deployed to watch tiny TCP/IP network simply. NetXRay improve the administrator to examine the network traffics and establish bottlenecks and potential issues. The simulator SSFNet is registered in BGP (border gateway protocol) [3] for analysis of domain popularly. For BGP simulation, Dimitropoulos et al. developed BGP++ ports that are used into NS-2 simulation 
atmosphere. Bro may be a network security monitor that is used in a top-level linguistics analysis at the end of application layer. Network flight recorder (NFR) provides valuable data concerning the expansion of the network, its usage patterns, potential misconfigurations, and more. Tcpreplay permits you to encapsulate and rejoin a true traffic. OMNet ++ is extensile, modular, component depends on $\mathrm{C}++$ simulation library and framework for fabrication of network simulators including wireless and wired networks.

\section{CRITERIA FOR IDS}

Intrusion detection systems are wide applied to prevent and reduce damage to information systems. The criteria which is applicable to intrusion detection system is delineated as follows.

Robustness - As a result of IDS is vulnerable to attack, survivability is an essential ability like redundancy, mobility, health checking, and dynamic recovery.

Potency - The IDS modules will execute the sniffing and analyze the code in time to discover the anomaly behaviors.

\begin{abstract}
Ability - The intrusion detection model doesn't depend on any special system, application environments, system vulnerabilities, or types of intrusions.
\end{abstract}

Measurability - IDS can scale sizes for various traffics that are increasing to avoid IDS services bottleneck.

Feedback - The feedback measures the system whether to discover intrusion and take bound actions automatically.

\section{DIFFERENT FORM OF SECURITY ATTACKS}

In traditional IDS there are 5 classes to tell different attacks from traditional traffics delineated as follows.

Login - Login activities give the users behavior information like last login, login frequencies, logout, login locations (remote host, network, port, terminal, workstation), location failure and password failure.

Execution - Execution activities describe the consequences of execution like read fail, read frequency, write frequency, write fail, delete frequency, create frequency execution denied, and execution frequency.

Session - Session (program) activities give the session resource usage information like time period, connections (resent rate, connection rejected, wrong size rate), I/O, resource exhaustion and CPU.

Exception - Exception conditions give the message of dealing error conditions without terminating execution of the program.

Connection activity - Connection activities give all connections messages and statements like SQL query and query timeout in SQL database. Furthermore the exploitation of system's vulnerabilities is split into eight parts of security attacks described as follows.
Attempted break-in - Attackers experiment to break in to a system by over flowing, dictionary attacks, port scan, and buffer attacks. Thus, there are some different abnormal phenomena like high percentage of long packets and password failures.

Prosperous break-in - Attackers easily break into the systems with success and use the normal username and password to attack other systems like backdoor attack, Worm, DDoS (distributed denial-of-service), Trojan horse and so on. When a penetrator logs into a system by unauthorized account and positive identification, many types of behaviors are totally different for the legitimate user depending on location, connection type, login time, most of time browsing directories, and executing system standing commands.

Penetration by legitimate user - Attacker act as legitimate user to attempts to access unauthorized programs or files by penetrating the security mechanisms within the operational system. User applies aggregation and inference to retrieve additional records than the standard to induce unauthorized data from database. In future back-end database servers will be the object of attacks by vulnerabilities of the online services like SQL injection [4].

Routing protocols - Routing protocols insecurity makes wireless networks adaptable to different types of outsider attacks like sinkhole attacks, Sybil attack, altered routing attack, and wormholes.

Privilege Escalation Attack [4] - In this kind of attack attacker behave like a regular user and logs in to the front end web server. After entering in to the front end web server he fires the admin level queries to get administrators data. Hijack Session Strike - This type of attack is generate in the front end web server side. Attackers are hijacking the legitimate users of sessions in front end web server side. This kind of attack is not generating in database environment.

Injection Attack - This kind of attack is not generating in web server. It can raise the problems in web server interface logic. Once the web server interface logic fails, problems are generating in database server. In SQL injection [4], some SQL queries are injected with the normal SQL queries to get data of different users.

Direct database attack [4] - A attacker can attack directly on database server or he/she can sign in to the front end web server and can take control over the front end web server and then fires queries to get data from the data base end server.

\section{INTRUSION DETECTION SYSTEM (IDS)}

In general intrusion detection system consists of 3 major element: resources, models, and techniques. Resources are protected within the object system like file systems, accounts, and system kernels. Models can recognize "normal" or "legitimate" behaviors of those resources. Techniques compare the particular system activities with the confirmed behavior models to detect "normal" or "intrusion". New technologies of computer networks became additional sophisticated. For intelligibility, intrusion detection systems are divided into three different types: IDS for database, traditional IDS, IDS for wireless network, delineate as follows. 


\subsection{Ids for Database}

In the classic three-tier model, data exchange and transmission between the front end web server and also the back end database server isn't separated. There are several types of attacks like privilege escalation attack, direct database attack, injection attack and hijack future session attack. Le et al. [4] conferred a double guard intrusion detection system to model the network reaction of different user sessions covering each web server and also the database server. Database should have the best level of preservation because of important information that stored in database. Therefore, outstanding analysis efforts have concentrate on database IDS and database firewalls [4]. These code [4] (software) works as a reverse proxy for database connections. In different words, web applications are going to be connected to database firewall rather than database server.

\subsection{Traditional Ids}

An intrusion detection System is systematized into hostdependent IDS, network-dependent IDS (NDIDS) and hybrid IDS. Host- dependent IDS depends on various operating systems. A network- dependent IDS [5] examines the network traffic to identify the intrusion activities over network. By analysis approaches, IDS is divided into 2 different types: one is misuse detection and second is anomaly detection.

Misuse detection systems [5] - Apply patterns of well-known attacks or system weakness to acknowledge Intrusion behaviors. The defect of misuse detection systems is unable to observe any future (unknown) intrusions until the corresponding attack signatures are intruded into the database server. Anomaly Detection - The crucial success of anomaly detection [5] depends on the model of traditional behavior. Most of systems experienced the selecting system options, e.g. CPU, I/O activities, TCP protocols. There are so many different varieties of techniques to get signatures as follows.

\subsubsection{Neural Network}

A neural network dependent intrusion detector for acknowledge of novel attacks [6] execute a ladder of back propagation in neural networks to watch selected areas of network behaviors. The neural networks are trained using normal operation of data that form traditional behavior models to detect attacks. A transfer control protocol is assume by the innovative (new) IDS like three-way connection installation handshake, the connection closing handshake, a sequence number matching, packet acknowledgment, and transmission and termination port choice.

\subsubsection{Data mining}

Signature Apriori [7], ID3 (iterative dichotomiser 3) [7] and k-nearest neighbor algorithm [8] all use the established historical profile to spot intrusions. There are total five components of Signature Sniffer: Signature miner, Packet detector, Signature Set, Associated Signature miner, and Rule Set. Packet detector monitors the packets from the network and sends them to Signature miner. Then Signature miner applies Signature Apriori to seek out the candidate signatures that have the greatest support. Signature set hold on those candidate signatures from Signature miner for more analysis. Depend on candidate signatures from Signature Set of different categories of signature, the associated signature miner totally concentrate on mining of associations of them. The candidate rules are going to be created and sent to Rule Set by the Associated Signature miner.

\subsection{Ids for Wireless Network}

Wireless devices could transfer data in between two or lot of points while not connected physically. Many wireless applications have more and more been applied to social networks, e-commerce, and industries. Normally there are many potential devastates to threat wireless networks. Many of us suggested new techniques and that are represented as follows.

\subsubsection{Wireless Sensor Networks (WSN)}

Because the wireless detector has become smaller and cost less, an outsized variety of sensors are deployed in a commercial ad hoc fashion to make a wireless sensor network for many environmental observance and different military applications. In general, detector nodes have restricted memory, computation resources, and power. Because of deployment of detector nodes in neglected and hostile environments, many individual sensors have vulnerabilities [4]. Therefore, intrusion detection is used to identify malicious or sudden intruders during a WSN. There are two major types of the WSN intrusion detection problem: using system elements to watch the security of a WSN and diagnoses related vulnerable sensors thus on make sure the correct network behavior and avoid a warning and second applying a monitoring or surveillance system to find a malicious intruder. Various styles of attacks in WSN are represented as follows.

Sinkhole attack - Attackers used the compromised nodes to draw in neighbor nodes to route them and make a metaphorical sink hole.

Spoofed, altered routing attack - Attackers produce routing loops, shorten transmitting routes by replaying routing data.

Flood attack - Attackers broadcast a very big amount of synchronizing packets to their neighbors.

Sybil attack - one node offers multiple originality to different nodes over the network.

Wormholes - Attackers penetrate messages accepted in one section of the network by a minimum latency link and answer the messages in a completely different part.

Selective forwarding - Attackers reject to forward certain messages.

\subsubsection{Security of Smart Phones}

Recently, sensible phones have considerably increased because completely different forms of connectivity are given, including GPRS, IEEE 802.11, GSM[9], Bluetooth, and HSPA. Nowadays smart mobile phone is become the target of attackers. Less non uniformity in operating system permits attackers to use simply one vulnerability to attack an oversized variety of various types of sensible phones. Nowadays, the number of OSs for sensible phones such as Symbian, Windows, Android, iPhone OS, OSs have increased. Because of additional users downloading various third-party application for sensible phones, more malwares (e.g. Backdoor, Trojan, rootkit, worm, virus, and botnet) [5] to attack various smart mobile phones can increase. 


\section{COMPARISON OF DIFFERENT INTRUSION DETECTION SYSTEMS}

Le et al. [4] compare the attack detections by different IDS. The table given below shows the comparison between different IDS. They used Snort as the network IDS in front of the webserver, and they used GreenSQL as the database IDS. They compare this two IDS with an IDS called doublegaurad [4]. The table shows that doublegaurad is efficient than this two ids for detecting attacks.

Table 1. Comparison of different IDS

\begin{tabular}{|l|l|l|l|}
\hline Attacks & Snort & GreenSQL & DoubleGuard \\
\hline $\begin{array}{l}\text { Privilege } \\
\text { Escalation } \\
\text { Attack }\end{array}$ & No & No & Yes \\
\hline $\begin{array}{l}\text { Session } \\
\text { Hijack Attack }\end{array}$ & Yes & No & Yes \\
\hline $\begin{array}{l}\text { Injection } \\
\text { Attack }\end{array}$ & No & Yes & Yes \\
\hline $\begin{array}{l}\text { Direct DB } \\
\text { Attack }\end{array}$ & No & No & Yes \\
\hline
\end{tabular}

\section{CHALLENGE FOR IDS ON DIFFERENT NETWORK}

Cloud computing and $\mathrm{P} 2 \mathrm{P}$ environment have become progressively attractive to the normal public. It is still a difficult issue to construct a secure computer networks and discover intruders. Several studies are proposed and are represented as follows.

\subsection{Cloud Computing Environment}

Cloud computing [10] is defined as the delivery of computing and storage capability as a service to end-users in a very miscellaneous community. Over the different kind computer and communication networks, computing providing different services with user's knowledge, software system and computation. Different species of cloud computing are as follows: i) Iaas for infrastructure as service, ii) SaaS for software as a service and iii) PaaS for platform as a service. The cloud computing has flourished and emerged because of the natural evolution and integration of advances in many area as well as web services, distributed computing, and service intended architectures. Several security risks have risen along with the uncountable edges like confidential information (financial and health data) emergence and loss of privacy in the cloud [11]. For secure knowledge sharing and protective user's privacy within the cloud, several studies are presented like privacy preserving public auditing, secure and dependable storage on cloud, file storage security maintenance etc.

\subsection{Unstructured P2P Environment}

$\mathrm{P} 2 \mathrm{P}$ systems are different from the actual network topology and designed at application layers. P2P systems which are distribute into structured and different unstructured peer-topeer kind of networks. Peer-to-peer networks which are structured, arrange according to determined criteria and various algorithms. Unstructured P2P [12] covering networks are divided systems without the centralized management. Every pc within the network is referred as a node and is allowed to access and allocate files and various peripherals without the essential of a central server. In alternative words, every peer might be a client or as a server. Via unstructured P2P networks, P2P worms [13] might infect shared files and propagate. Depends on the actual situation of P2P users, a delayed SEIRS (susceptible, evident, infectious, removed, and susceptible) epidemic model with death, offline and on-line rate was made. A workload-driven simulation framework characterizes three kinds of non-scanning worms like passive worm, proactive worm, and reactive worm. So as to reveal worms spreading capability, several analysis models [14] were proposed such as neuro fuzzy engine and fuzzy rules.

\section{CONCLUSION}

Because high-speed backbones and local area networks (LAN) provide the end user with bandwidths, high speed, the internet has become a remarkable infrastructure for governments, different companies, and several terminating users. New technologies of computer networks have additionally become a lot of sophisticated, including wireless devices (e.g. smart phone, wireless sensor), cloud computing, and web applications. Thus, it's tough to fulfil the protection demands for all networks by IDS. Because wireless devices became smaller and price less, a large number of wireless devices are deployed in ad hoc fashion to form wireless networks. Several individual wireless devices are vulnerable in unsupervised and hostile environments. Via multitier internet applications and wireless devices (e.g. smart phone ), web-delivered services and applications have considerably magnified, including banking, travel, invest, shopping, and social networking. The back-end data should have the better level of protection to prevent valuable info from uncertified access. On networks trends, cloud computing and P2P environment are the challenge to IDS. Nowadays, many enterprises created two or more IDS products to detect intrusions more efficiently. However, those of IDS products are independent and a hardly any of their functions are overlay. The way to integrate and manage totally different varieties of IDSs is necessary problems to lower the cost.

\section{REFERENCES}

[1] The Bro Network Security Monitor. [Online]. Available: http://bro-ids.org.

[2] Network Flight Recorder. [Online]. Available: http://www.checkpoint.com/ corporate/nfr/index.html.

[3] X. A. Dimitropoulos and G. F. Riley, "Creating realistic BGP models," in Proc. of the 11th IEEE/ACM Int Symposium on Modeling, Analysis and Simulation of Computer and Telecommunication Systems, Orlando, 2003, pp. 64-70.

[4] M. Le, A. Stavrou, and B. B. H. Kang, "Double guard: detecting intrusions in multitier web applications," IEEE Trans. on Dependable and Secure Computer, vol. 9, no. 4, pp. 512-525, 2012.

[5] Y.-J. Lee, Y.-R. Yeh, and Y.-C. F. Wang, "Anomaly detection via online over-sampling principal component analysis," IEEE Trans. on Knowledge and Data Engineering, doi: 09/TKDE.2012.99, 2012.

[6] M. Mohajerani, A. Moeini, and M. Kianie, "NFIDS: A neuro-fuzzy intrusion detection system," in Proc. of the 10th IEEE Int. Conf. on Electronics, Circuits and Systems, Sharjah, 2003, pp. 348-351. 
[7] Y. Wang, W. Fu, and D. P. Agrawal, "Gaussian versus uniform distribution for intrusion detection in wireless sensor networks," IEEE Trans. on Parallel and Distributed Systems, doi: 09/TPDS.2012.105, 2012.

[8] K. Ilgun, R. A. Kemmerer, and P. A. Porras, "State transition analysis: a rule-based intrusion detection approach," IEEE Trans. on Software Engineering, vol. 21, no. 3, pp. 181-199, 1995.

[9] C.-C. Lee, M.-S. Hwang, and W.-P. Yang, "Extension of authentication protocol for GSM," IEE Proc. Communications, vol. 150, no. 2, pp. 91-95, Apr. 2003.

[10] H.-Y. Lin and W.-G. Tzeng, "A secure erasure code based cloud storage system with secure data forwarding," IEEE Trans. on Parallel and Distributed Systems, vol. 23, no. 6, pp. 995-1003, 2012.

[11] R. Sanchez, F. Almenares, P. Arias, D. Diaz-Sanchez, and A. Marin, "Enhancing privacy and dynamic federation in IdM for consumer cloud computing," IEEE Trans. on Consumer Electronics, vol. 58, no. 1, pp. 95$103,2012$.

[12] F. Wang, Y. Zhang, and J. Ma, "Modelling and analyzing passive worms over unstructured peer-to-peer networks," Int.Journal of Network Security, vol. 11, no. 1, pp. 39-45, 2010.

[13] C.-Y. Ho, Y.-C. Lai, I-W. Chen, F.-Y. Wang, and W.-H. Tai, "Statistical analysis of false positives and faluse negatives from real traffic with intrusion detection/prevention systems," IEEE Communications Magazine, vol. 50, no. 3, pp. 146-154, 2012.

[14] M. Mohajerani, A. Moeini, and M. Kianie, "NFIDS: A neuro-fuzzy intrusion detection system," in Proc. of the 10th IEEE Int. Conf. on Electronics, Circuits and Systems, Sharjah, 2003, pp. 348-351. 